Mode oscill ati on and har moni $c$ di st orti ons associ at ed wi th si nusoi dal modul at i on of sem conduct or I aser s

\begin{tabular}{|l|l|}
\hline 著者 & Ahned Moust af a, Yanda M nor u \\
\hline $\begin{array}{l}\text { j our nal or } \\
\text { publ i cat i on ti t l e }\end{array}$ & Eur opean Physi cal Jour nal D \\
\hline vol une & 66 \\
\hline number & 9 \\
\hline page r ange & 246 \\
\hline year & $2012-09-01$ \\
\hline URL & ht t p: //hdl . handl e. net /2297/32821 \\
\hline
\end{tabular}




\title{
Mode Oscillation and Harmonic Distortions Associated with Sinusoidal Modulation of Semiconductor Lasers
}

\author{
Moustafa Ahmed ${ }^{1}$ and Minoru Yamada ${ }^{2}$ \\ ${ }^{1}$ Department of Physics, Faculty of Science, King Abdulaziz University, Jeddah, Saudi Arabia. \\ Tel: +966540791790 Fax: +96626951106 \\ Email: mostafa.hafez@ science.miniauniv.edu.eg \\ ${ }^{2}$ Division of Electrical Engineering and Computer Science, Graduate School of Natural Science and \\ Technology, Kanazawa University, Kakuma-machi, Kanazawa 920-1192, Japan. \\ Email: myamada@t.kanazawa-u.ac.jp
}

Short title: Mode oscillation and harmonic distortion in modulated laser diodes

\begin{abstract}
This paper investigates mode dynamics, operation characteristics and signal distortions associated with sinusoidal modulation of semiconductor lasers. The study is based on intensive integrations of the multimode rate equation model of semiconductor lasers over wide ranges of the modulation frequency and depth. The rate equations take into account both spectral symmetric and asymmetric suppressions of modal gain. The higher harmonic distortions as well as the half harmonic distortion associated with the period doubling effect are investigated. The study is applied to both cases of single-mode and multimode oscillations of the non-modulated laser. The obtained results showed that the modulated signal has six distinct waveforms depending on the modulation conditions; three types have continuous periodic waveforms and the others have periodic pulsing waveforms. The modulated laser is found to oscillate in a single mode under weak modulation where the modulated signal is continuous, whereas the pulsing signals are associated with multimode oscillation. The higher harmonic distortions of single-mode laser are lower than those of two-mode lasers, and become serious at modulation frequencies around the relaxation oscillation frequency. These distortions are highest when the laser output is pulsating and the pulses are superposed by relaxation oscillations.
\end{abstract}




\section{Introduction}

Semiconductor lasers are key radiation sources in digital and analog optical applications. The latter include RF fiber links, which are increasingly used for the distribution of radio frequency signals in mobile communication networks and WLANs [1]. Laser diodes oscillating in single mode are preferable for these fiber links applications as they achieve lower noise levels, narrower spectral lines and lower harmonic distortions. In actual fact, single-mode operation is a matter of approximations. This is because even when the side mode suppression ratio (SMSR) is as high as 100, competition among the oscillating modes in the laser cavity may cause unstable dynamics such as jittering single mode or multimode hopping, depending on the operation conditions as well as the structure geometry of the cavity $[2,3]$. The laser may then oscillate simultaneously in more than one mode which increases the noise level [4] and enhances the effect of fiber dispersion in the form of mode partition [5]. The mode competition originates from the cross-modal gain suppression, which has two spectral types; namely, symmetric gain suppression (SGS) and asymmetric gain suppression (ASGS) [6,7]. The former causes symmetric suppression of modal gain with respect to the central mode of the gain spectrum. AGS works to increase the gain of modes on the longer wavelength side of the central mode while decreasing the gain of the modes on the shorter side [7]. AGS is a source of mode jumping in single mode lasers [3] and multimode hopping in long-wavelength lasers [8].

Modulation of the laser diode by RF signals in fiber links is also a nonlinear effect especially under large-signal modulation. This modulation has been shown to induce inconsistence of the timedomain characteristics of the modulated laser signal with those of the modulating current signal. The sinusoidally modulated laser signal happens to be continuous, but not sinusoidal, or pulsing, and may have period doubling $[9,10]$. These forms have different noise levels, that may be comparable to the quantum noise level or may be $-40 \mathrm{~dB} / \mathrm{Hz}$ higher $[10,11]$. On the other hand, sinusoidal modulation was found in experiments to convert the single mode oscillation of a laser into multimode oscillation under strong modulation [12]. Detailed investigations of mode competition and its influence on modal oscillations of 
the both single and multimode lasers are then needful for more understanding the physics of the semiconductor laser and for controlling its performance under modulation.

Coupled with laser nonlinearities, analog modulation induces also distortions of the optical signal, which limits the maximum usable bandwidth of the fiber link [13], especially when the signal amplitude is large [14]. Because of this signal distortion, there is inevitably some modulation power at the harmonics of the modulation frequency and, consequently, degradation of the output signal quality [1]. Halfharmonic distortion may also arise when the modulated signal has period doubling. Nonlinear distortion in semiconductor lasers has been theoretically analyzed since 1970s [15]. It has been shown that nonlinear distortion becomes extremely serious at modulation frequencies around the relaxation oscillation frequency of the laser [15]. Nonlinear distortion of various laser systems under intensity modulation was experimentally and theoretically confirmed by Y. Kam and A. Yariv [16]. Studying the harmonic distortions associated with mode competition under modulation is critical for evaluation of the modulation performance of semiconductor lasers.

In this paper, we introduce large-signal analyses of mode competition of semiconductor lasers subjected to sinusoidal modulation. The associated higher and half harmonic distortions of the modulated signal are investigated over wide ranges of the modulation frequency and depth. Both cases of nearly single mode and two mode oscillations of the non-modulated laser are considered and comparison of the modulation characteristics of the laser under both cases is given. The analyses are bases on intensive numerical integration of multimode rate equations of the laser including both SGS and AGS of modal gain. The obtained results show that the modulated laser waveform is continuous periodic signal (CPS), continuous periodic signal with relaxation oscillations (CPSRO), continuous periodic signal with period doubling (CPSPD), periodic pulse (PP), periodic pulse with relaxation oscillations (PPRO) or periodic pulse with period doubling (PPPD). The laser is proved to oscillate in single mode when the signal is continuous, whereas the pulsing signals are characterized by multimode oscillation. This range of the modulation depth of modulated single-mode oscillation is smaller for the two-mode non-modulated laser than for the single-mode laser. Modulation of the two-mode laser is associated with wider operating 
ranges of the pulsing types than those of the nearly single-mode laser. The higher harmonic distortions of nearly single-mode laser are lower than those of two-mode lasers, and become serious at modulation frequencies around the relaxation oscillation frequency. These distortions are highest when the laser output is pulsating and the pulses are superposed by relaxation oscillations.

The paper is organized as follows. In the following section, the simulation model of mode competition dynamics and harmonic distortion is introduced. Section 3 introduces the calculation procedures of modulation characteristics of the laser. Section 4 presents the simulation results of mode competition dynamics and distortion. Conclusions of the present work appear in section 5 .

\section{Theoretical model of analysis}

The present analysis of mode dynamics of semiconductor lasers under modulation is based on the following multimode rate equation model of the injected carrier number $N(t)$ and modal photon number $S_{p}(t)$ with $p=0, \pm 1, \pm 2, \ldots M$ :

$$
\begin{aligned}
& \frac{d N}{d t}=\frac{1}{e} I(t)-\sum_{p} A_{P} S_{p}-\frac{N}{\tau_{s}} \\
& \frac{d S_{p}}{d t}=\left(G-G_{p}\right) S_{p}+\frac{a \xi}{V} \frac{N}{1+\left(2 \frac{\lambda_{p}-\lambda_{p e a k}}{\delta \lambda}\right)^{2}}
\end{aligned}
$$

where $G_{p}$ is the gain of mode $p$ whose wavelength is $\lambda_{p}$. $G_{p}$ is defined such that the suppression by mode $p$ itself and both SGS and AGS by other modes $q \neq p$ are taken into account [2,3],

$$
G_{p}=A_{p}-S_{p}-\sum_{q \neq p}\left\lfloor D_{p(q)}+H_{p(q)}\right\rfloor S_{q}
$$

$A_{p}$ is the linear gain, $B$ is the coefficient of self-suppression, and $D_{p(q)}$ and $H_{p(q)}$ are coefficients of SGS and AGS, respectively. These coefficients are given by $[2,3]$ :

$$
A_{p}=\frac{a \xi}{V}\left[N-N_{g}-b V\left(\lambda_{p}-\lambda_{\text {peak }}\right)^{2}\right]
$$




$$
\begin{aligned}
B & =\frac{9}{2} \frac{\pi c}{\varepsilon_{0} n_{D}^{2} \hbar \lambda_{0}}\left(\frac{\xi \tau_{\text {in }}}{V}\right)^{2} a\left|R_{c v}\right|^{2}\left(N-N_{s}\right) \\
D_{p(q)} & =\frac{4}{3} \frac{B}{\left.2 \pi c \tau_{i n} / \lambda_{p}^{2}\right)\left(\lambda_{p}-\lambda_{q}\right)^{2}+1} \\
H_{p(q)} & =\frac{3}{8 \pi}\left(\frac{a \xi}{V}\right)^{2}\left(N-N_{g}\right) \frac{\alpha \lambda_{p}^{2}}{\lambda_{q}-\lambda_{p}}
\end{aligned}
$$

In Eq. (4), $a$ is the differential gain, $\xi$ is the field confinement factor, $\lambda_{\text {peak }}$ is the wavelength at the peak of the linear gain spectrum $A_{p}$ whose width is $b$, and $N_{g}$ is the electron number at transparency. In the nonlinear gain equations (5)-(7), $\tau_{i n}$ is the intraband relaxation time, $R_{c v}$ is the dipole moment, $N_{s}$ is an electron number characterizing $B$, and $\alpha$ is the linewidth enhancement factor. Remaining parameters are: $I$ is the injection current, $n_{D}$ is the refractive index of the laser cavity whose volume is $V$ and length is $L_{D}, e$ is the electron charge, $\tau_{s}$ is the electron lifetime by spontaneous emission, and $\bar{N}$ is the time-average value of $N(t)$. The last term in Eq. (2) represents inclusion of the spontaneous emission into the lasing mode [2].

The central mode $p=0$ with wavelength $\lambda_{\rho}$ is assumed to lie at the peak of the gain spectrum, and the wavelength of the other modes is defined as:

$$
\lambda_{p}=\lambda_{0}+p \Delta \lambda=0, \pm 1, \pm 2, \pm 3, \ldots . M
$$

where $\Delta \lambda=\lambda_{0}^{2} / n_{D} L_{D}$ is the modal wavelength separation. The mode index $p$ is positive for modes lying on the long wavelength side of the central mode, i.e., $\lambda_{p}>\lambda_{0}$, and is negative for modes with shorter wavelengths, $\lambda_{p}<\lambda_{0}$. Equation (7) indicates that the AGS coefficient $H_{p(q)}$ is inversely proportional to $\lambda_{q}-$ $\lambda_{p}$. Therefore, AGS of mode $p$ works to suppress gain of modes $q \neq p$ with shorter wavelength but enhance gain of the modes with longer wavelengths.

The sinusoidal modulation is included in the rate equations by representing the time-varying current $I(t)$ in rate equation (1) of $N(t)$ by the following sinusoidal form 


$$
I(t)=I_{b}\left\{1+m \cos \left(2 \pi f_{m} t\right)\right\}
$$

where $I_{b}$ is the bias current, $m$ is the modulation depth and $f_{m}$ is the modulation frequency.

The frequency content of the modulated laser signal is determined by the frequency power spectrum $S_{f}$ of the fast Fourier transform (FFT) of the temporal trajectory of $S(t)$. This FFT power spectrum is calculated over a finite period $T$ as

$$
S_{f}=\frac{1}{T}\left|\int_{0}^{T} S(t) e^{-j \Omega \tau} d \tau\right|^{2}
$$

where $f=\Omega / 2 \pi$ is the Fourier frequency. The relative amplitudes $\left(a_{n}\right)$ of the peaks of this FFT power spectrum $S_{f}$ and their frequency positions are used to evaluate the harmonic distortions associated with the signal modulation. The $n^{\text {th }}$ order harmonic distortion (nHD) is evaluated in decibels $(d B)$ as the ratio of the amplitude $a_{n}$ of the $n^{\text {th }}$ harmonic to that of the first (fundamental) harmonic [17]:

$$
\mathrm{nHD}(\mathrm{dB})=20 \log _{10}\left(\frac{a_{n}}{a_{1}}\right)
$$

When the modulated signal has period doubling effect, the half harmonic distortion (HHD) is determined in similar fashion in terms of the amplitude $a_{1 / 2}$ of the peak at the half harmonic frequency $f=f_{m} / 2$.

\section{Procedures of Numerical Calculation}

The system of rate equations (1) and (2) are solved numerically by the fourth order Runge-Kutta method using an integration step of $\Delta t=10 \mathrm{ps}$. A large number of 15 modes $(M=7)$ are counted in the calculations to ensure accuracy of analyzing the mode competition dynamics. The laser is assumed to be biased above threshold to neglect contribution of the noisy spontaneous emission to the laser output. 850-nm AlGaAs laser diodes are assumed for the present calculations. Typical values of the parameters of this laser are listed in table 1 . The relaxation frequency $f_{r}$ of the laser is determined approximately from the small-signal approach as [11] 


$$
f_{r} \approx \frac{1}{2 \pi} \sqrt{\left(\frac{a \xi}{V}\right)\left[\frac{a \xi \tau_{e}}{e V}\left(I_{b}-I_{g}\right)+B \frac{I_{b}-I_{t h}}{e G_{t h}}\right] \frac{I_{b}-I_{t h}}{e G_{t h}}}
$$

where $I_{g}=e N_{g} / \tau_{s}$ is the current at transparency, and $\mathrm{S}_{\mathrm{b}}$ is the bias component of the total photon number $S(t)$ and is evaluated via the equation

$$
S_{b}=\frac{I_{b}-I_{t h}}{e G_{t h}}
$$

with $I_{t h}$ being the threshold current. The output spectrum of the laser that determines SMSR and reflects the modal oscillation is calculated by averaging the temporal modal photon numbers $S_{p}(t)$ and constructing a bar plotting of these average values $\overline{S_{p}}$ versus the mode index $p$. SMSR of the multimode oscillation is evaluated as the ratio of the average value of the dominant mode to that of the strongest side mode. In the present work, the condition of single mode oscillation is defined as SMSR > 50. The FFT power spectrum $S_{f}$ is calculated numerically via Eq. (10) as

$$
S_{f}=\frac{1}{T}\left|\int_{0}^{T} S(t) e^{-j \Omega \tau} d \tau\right|^{2}=\sqrt{\frac{\Delta t}{T}}|F F T(S(t))|
$$

\section{Results and Discussion}

\subsection{Relaxation oscillations of non-modulated laser}

Mode competition happens to induce single mode oscillation or oscillation in more than one mode of the semiconductor laser whose geometry support multimode oscillations, such as lasers with Fapry-Perot cavities or VCSELs. In such cases, single mode oscillation is virtual, and the laser is said to be nearly single mode laser. The dynamics of such lasers depend on the operating conditions. Figures 1(a) and (b) plot the laser transients of two-distinct operational states of the laser; namely, nearly single-mode oscillation $\left(I=3.0 I_{t h}\right)$ and two-mode oscillation $\left(I=2.01 I_{t h}\right)$, respectively. The figures plot the transient trajectories of photon number $S_{p}(t)$ of the strongest oscillating modes and the total photon number $S(t)$. The figures show the phenomenon of relaxation oscillations of the intensities of both the oscillating modes and the total output that follow the turn-on delay effect. The temporal competition among the oscillating 
modes to attain the highest gain and contain the laser output is clear up to $t=6 \mathrm{~ns}$ before the laser output attains steady state values. In general, the competing modes are those lying on the longer wavelength side of the central mode $p=0$, because these modes are supported by higher modal gain due to the AGS that works to increase the gain of modes with longer wavelengths while decreasing the gain of modes with shorter wavelengths. Figure 1(a) shows that mode $p=+2$ becomes the dominant mode and the laser oscillates dominantly in this mode. On the other hand, figure 1(b) indicates that both modes $p=+1$ and +2 dominates the laser oscillations and carry most of the laser output.

Figure 2 compares mode competition of both cases of nearly single mode oscillation (figure 1(a)) and two-mode oscillation (figure 1(b)) in terms of the spectra of linear gain $A_{p}$ and suppressed gain $G_{p}$ of the oscillating modes. The figure shows that the linear gain $A_{p}$ is higher when $I=3.0 I_{t h}$ than when $I=2.01 I_{t h}$ due to the higher injection level of the laser. The figure indicates the asymmetric character of the spectra of suppressed gain $G_{p}$ around the central mode $p=0$ due to AGS. In the vicinity of the central modes, AGS works to increase the gain of longer wavelengths while decreasing the gain of shorter wavelengths. The degree of asymmetry is higher when $I=3.0 I_{t h}$ than when $I=3.0 I_{t h}$. When $I=3.0 I_{t h}$, the gain difference between the strongest mode $p=+2$ and next strongest mode $p=+3$ is $G_{+2}-G_{+3}=0.0029 G_{t h}$, which results in the single mode, $p=+2$, oscillation seen in figure 1(a). On the other hand, this gain difference is as small as $G_{+1}-G_{+2}=0.000045 G_{t h}$, which results in the two-mode oscillation of figure 1(b).

In the following subsections, we present the dynamics and modal oscillation of the laser at these two distinct operational states when the laser is subjected to sinusoidal modulation.

\subsection{Types of laser waveforms and modal oscillation under modulation}

The present sinusoidal modulation of the semiconductor laser generates six distinct forms of the output signal. In this subsection, we characterize these signal types in terms of the signal waveforms of the modes, the total output of mode intensities, the FFT frequency power spectra and the associated harmonic distortion. The characterization is presented in figure 3 for the case of nearly single-mode oscillation when $I_{b}=3 I_{t h}$. The left hand side column plots the temporal trajectory of the total photon number $S(t)$, the next 
column plots the transients of the strongest three oscillating modes, the third column plots the output spectrum of mode intensities, while the right hand side column plots the FFT power spectrum $S_{f}$ of the investigated types of the modulated waveform.

Figures 3(a) - (d) characterize the case of CPS in which $S(t)$ varies continuously and periodically with the time variation, as shown in figure 3(a). The figures corresponds to $f_{m}=f_{r}$ and $m=0.1$. This time variation becomes sinusoidal under very weak modulation $(m<0.05)$. This type of signals was observed in experiments by Henery et al. [18] and simulated by Ahmed and El-Lafi [12]. In this case, the laser output is mainly contained in mode $p=+2(\mathrm{SMSR}=338)$ as indicated in the output spectrum of figure 3(c). Figure 3(b) shows the sever mode competition among modes $p=0,+2$ and +3 resulting in multimode oscillation up to $t=4 \mathrm{~ns}$, after which mode $p=+2$ attains the maximum gain and dominates laser oscillation. The FFT power spectrum $S_{f}$ in figure $3(\mathrm{~d})$ is characterized by a sharp peak at $f_{m}$ and a weak peak at $2 f_{m}$. The associated $2^{\text {nd }}$ harmonic distortion is $2 \mathrm{HD}=-1.42 \mathrm{~dB}$, while the $3^{\text {rd }}$ harmonic distortion is $3 \mathrm{HD}=-35.3 \mathrm{~dB}$.

With the increase in the modulation depth $m$ in the region of low-frequency modulation, the gain decreases further under the threshold level $G_{t h}$ around the valleys of the modulating current signal $I(t)$. This effect results in the CPSRO type as given in the waveform of figure 3(e). The shown signal corresponds to $f_{m}=0.2 f_{r}$ with $m=0.4$, and was observed also in the experiments of Henery et al. [18] and predicted by Ahmed and El_Lafi in single-mode lasers [11]. In this case also, the laser output is mainly contained in mode $p=+2$ (SMSR=280) as indicated in the output spectrum of figure 3(g). Similar to figure 3(c) of the CPS type, figure 3(f) shows that the mode competition is strong among modes $p=0,+2$ and +3 resulting in multimode oscillation up to $t=4 \mathrm{~ns}$, after which mode $p=+2$ attains the maximum gain and dominates laser oscillation. The FFT spectrum $S_{f}$ in figure 3(h) is also characterized by a sharp peak at $f_{m}$ and a weak peak at $2 f_{m}$. Both $2 \mathrm{HD}$ and $3 \mathrm{HD}$ are $-26.4 \mathrm{~dB}$ and $-28.8 \mathrm{~dB}$, respectively.

Under high modulation frequencies, the continuously varying laser intensity exhibits period doubling, where the modal gain goes far below $G_{t h}$. Therefore, the effect of the turn-on delay is pronounced in the time variation of $S(t)$ in the form of two unequal peaks. This CPSPD type is characterized in figures 3(i) - (1), which correspond to $f_{m}=1.4 f_{r}$ and $m=1.3$. Figure 3(i) shows that the total 
photon number $S(t)$ has two peaks of different peaks in every two successive periods. The separation of each two successive similar peaks is equal to $2 T_{m}=f_{m} / 2$. In this type, the laser still oscillates in single mode, $p=+2$, but with lower value of SMSR $=56.4$. This is illustrated in the output and gain spectra of figure $3(\mathrm{k})$. In this case, figure $3(\mathrm{j})$ shows that the transient competition among the oscillating modes $p=-$ $2,+2$ and +3 continues for longer times than the cases of CPS and CPSRO and the oscillation amplitude of the dominant mode $p=+2$ is much larger. The irregularity of the CPSPD is indicated in the FFT frequency spectrum figure $3(1)$ in terms of the pronounced peaks at $f_{m}$ and $f_{m} / 2$ as well as the higher harmonics. Therefore this type has half harmonic distortion (HHD) of $1.37 \mathrm{~dB}$, which is much higher than $2 \mathrm{HD}=-$ $21.5 \mathrm{~dB}$ and $3 \mathrm{HD}=-35 \mathrm{~dB}$.

Under rather strong modulation, the continuous variation of the laser Intensity converts to pulsation due to stronger drop of gain under $G_{t h}$ during the valleys of $I(t)$. The uniform time variation is in the form of PPs, which is characterized in figure $3(\mathrm{~m})-(\mathrm{p})$. In this case, $f_{m}=f_{r}$ and $m=1.2$. Figure $3(\mathrm{~m})$ shows that $S(t)$ exhibits uniform PPs. In this case, the drop of gain under threshold is smaller than the value of the CPSPD type. When the pulse is too short, this type corresponds to the spike generation predicted by Lee et al. [19]. Contrary to the cases of continuous variation of $S(t)$, the laser output is contained in several modes $p=0,+2$ and +3 , as shown in figure 3(n), which indicates comparable values of the oscillation amplitudes of the strongest modes. This multimode oscillation is indicated also in the multimode spectrum of figure 3(o) with SMSR=5.6. Figure 3(p) plots the FFT spectrum $S_{f}$, which has peaks at $f_{m}$ and the higher harmonics. In this case $2 \mathrm{HD}=-0.5 \mathrm{~dB}$ and $3 \mathrm{HD}=-8.4 \mathrm{~dB}$ which are much higher than those of the CPS type.

Under low-modulation frequencies where the modulation period $T_{m}$ is long enough for the relaxation oscillations to appear, the periodic pulsation is superposed by relaxation oscillations when strong modulation enhances the turn-on effect. Figure 3(q) plots the time characteristics of this CPSRO type when $f_{m}=0.1 f_{r}$ and $m=1.2$. In this case the laser output and the modal gain spectrum are homogeneously distributed among the oscillating modes, and the output spectrum is of a typical multimode type, as shown in figure 3(s). Figure 3(r) indicates that the strongest oscillating modes $\mathrm{p}=0,+1$ 
and +2 have almost equal values during the pulsations of the modes. The FFT frequency spectrum of figure 3(t) indicates a pronounced peak at $f_{m}$ and smaller peaks at $2 f_{m}$ and $3 f_{m}$. The corresponding harmonic distortions are $2 \mathrm{HD}=-6.74 \mathrm{~dB}$ and $3 \mathrm{HD}=-13.7 \mathrm{~dB}$, which are higher than those of the CPSRO type.

The periodic pulsation happens also to exhibit period doubling under strong modulation with high modulation frequencies. This PPPD type is characterized in figure 3(u) $-(\mathrm{x})$ when $m=1.3$ and $f_{m}=f_{r}$. Figure 3(u) shows that the pulses of $S(t)$ have a pulse every two successive periods, that is, the peak separation is $2 T_{m}=f_{m} / 2$. Contrary to the CPSPD type, this type is characterized by spectral homogenous distribution of modal gain and typical multimode output as shown in figure $3(\mathrm{w})$. The period doubling dynamics were observed in experiments by Chusseau et al. [9] and Henery et al. [18] and predicted in theory [11,21]. Similar to figure 3(r) of the CPSRO type, figure 3(v) indicates almost comparable values of the stongest oscillating modes $p=0,+1$ and +2 . Figure $3(\mathrm{x})$ reflects the harmonic distortions associated with this type of the modulated signal. The values of $\mathrm{HHD}, 2 \mathrm{HD}$ and $3 \mathrm{HD}$ are $-1.56 \mathrm{~dB},-11.6 \mathrm{~dB}$ and $-11.4 \mathrm{~dB}$, respectively.

\section{3. ( $m$ versus $\left.f_{m}\right)$ diagram of modulation dynamics and modal oscillation}

In this subsection, we explore the modulation conditions that correspond to both uniform laser output with low harmonic distortions, and non-uniform output in order to avoid the associated high signal distortions. The laser dynamics are simulated over wide ranges of the modulation depth, $m=0.01-1.5$, and modulation frequency, $f_{m}=0.05 f_{r}-f_{3 d B}$, where $f_{3 d B} \approx 1.6 f_{r}$ [22] is the modulation bandwidth frequency. The modulation type corresponding to each modulation condition $\left(m, f_{m}\right)$ is then decided as discussed above. The modulation parameters, $m$ and $f_{m}$, corresponding to each of the investigated modulation types are mapped in the ( $m$ versus $f_{m}$ ) diagram of figure 4 . Therefore, each domain in the diagram encloses modulation points ( $m$ versus $f_{m}$ ) that reveal the modulated laser output of designated type. Figure 4 includes also a contour plotting of SMSR as a function of $m$ and $f_{m}$, which points out the modal oscillation of the laser over the entire ranges of modulation conditions.

The figure shows that at each value of $f_{m}$, the CPS type dominates the range of low values of $m$. The range of $m$ in the region of the CPS type increases with the increase in $f_{m}$. At a given value of $m$ 
within this CPS region, the signal amplitude increases with the increase in $f_{m}$ revealing a peak around the relaxation frequency $f_{r}$ and then decreases with further increase in $f_{m}$. This behavior fits the famous smallsignal modulation response [23]. Up to $f_{m}=0.4 f_{r}$, the increase in $m$ converts the CPS type into the CPSRO type and then to the PPRO type. Within the operating region of the PPRO type, the degree of this nonuniformity increases with the increase in $m$. At rather high values of $0.4<f_{m} / f_{m}<1$, strengthening the modulation converts the CPS type directly into the discontinuous PP type, which agrees with the predictions of Kao and Lin [24]. Therefore, it can be decided that this range of $f_{m}$ corresponds to uniform and periodic laser output; it is either CPS or PP regardless of the relevant range of $m$. At a given value of $f_{m}$, the pulse width decreases with the increase in $m$. At higher values of $f_{m}$, when $m$ increases beyond 1.2, the period doubling effect appears; it starts with the CPSPD type and then converts into the PPPD type. Other forms of the PPPD include one pulse (with equal or little unequal peaks) every two successive periods. The degree of this non-uniformity increases with the increase in $m$.

The contour plot of SMSR in figure 4 shows that the laser almost operates in single mode, $p=+2$, when the signal exhibits continuous variation with time, whether the signal has the CPS, CPSRO or CPSPD type. However, near the boundaries between the continuous variation and pulsation, the value of SMSR decreases and the operation converts to multimode. Within the operating regions of pulsing outputs, SMSR decreases remarkably with the increase in $m$, which is associated with an increase in the width of the spectrum. These results are in good agreement with the experimental observations by Lau et al. [12] that under strong modulation, the single-mode lasing spectrum breaks into multimode oscillation and the envelope width of the spectrum increases rapidly with the increase in the modulation depth.

It is interesting to compare the investigated modulation dynamics with those obtained when $I_{b}=2.01 I_{t h}$ at which the non-modulated laser oscillate in two modes as given in figure 1(b). Figure 5 plots the ( $m$ vs. $\left.f_{m}\right)$ diagram at this bias level. The figure indicates almost similar modulation characteristics in figure 4 of the nearly single-mode oscillation of the non-modulated laser when $I_{b}=3.0 I_{t h}$. By comparing this diagram and the overlying plot contour of SMSR with those of figure 4, we can indicate the following results of comparison results on the modulation characteristics between the single and two-mode lasers. 
The sinusoidal modulation converts the two-mode oscillation into oscillation in single mode over the low ranges of $m$ (weak modulation). This range of $m$ of modulated single-mode oscillation, however, is smaller than that of figure 4 when $I_{b}=3.0 I_{t h}$. For example when $f_{m}=f_{r}$, the single mode oscillation continues up to $m=0.2$ for $I_{b}=2.01 I_{t h}$ whereas it is up to $m=0.6$ for $I_{b}=3.0 I_{t h}$. The modulation at $I_{b}=2.01 I_{t h}$ is also associated with enlargement of the operating ranges of $m$ of the pulsing types at expense of the operating ranges of $m$ of the continuously varying signals. The reduction is significant in the operating ranges of the CPS type. The CPSRO type is seen to extend over higher modulation frequencies, up to $f_{m}=0.6 f_{r}$, whereas this upper modulation frequency is $f_{m}=0.6 f_{r}$ when $I_{b}=3.0 I_{t h}$.

\subsection{Harmonic distortion}

Harmonic distortion is one of the sinusoidal modulation properties that limit application of semiconductor lasers in radio fiber links [3]. In this subsection we investigate dependence of the higher harmonic distortions, 2HD and 3HD, as well as the half-harmonic distortion HHD, on the modulation conditions $f_{m}$ and $m$. Obviously HHD is enhanced when the modulated signal exhibits period doubling. Figures 6(a) (c) plot dependencies of these harmonic distortions for the case of nearly single-mode oscillation on the modulation depth $m$ at three values of the modulation frequency, $f_{m}=0.1 f_{r}, f_{r}$ and $1.4 f_{r}$, respectively. The figures show that $2 \mathrm{HD}$ is higher than 3HD in general over the relevant ranges of $m$ and $f_{m}$. Figure 5(a) shows that in the region of low-modulation frequencies, $2 \mathrm{HD}$ increases with the increase of $m$. 3HD increase also with the increase in $m$ up to $m=0.8$, above which $3 \mathrm{HD}$ decreases little. These distortions are highest within the operating range of the PPRO type, lower within the range of the CPSRO type and lowest within the operating range of the CPS type. The increase of $2 \mathrm{HD}$ in the regime of the PPRO type is manifestation of an increase in the degree of non-uniformity of the PPRO signals.

Figure 6(b) shows that when $f_{m}=f_{r}, 2 \mathrm{HD}$ and 3HD increase little with the increase of $m$ within the operating ranges of the CPS and PP types. In the operating range of the PPPD type, 2HD and 3HD decrease with the increase of $m$, while the half-harmonic distortion HHD increases to large values. The figure indicates also that the ranges of $2 \mathrm{HD}$ and $3 \mathrm{HD}$ in this case are higher than those of the low- 
frequency modulation, $f_{m}=0.1 f_{m}$, of figure 6 (a). In the region of higher modulation frequency, $f_{m}=1.4 f_{m}$, figure 6(c) shows that $2 \mathrm{HD}$ and $3 \mathrm{HD}$ increase with the increase of $m$ except within the operating range of the CPSPD region. The values of 2HD characterizing the CPS type are smaller than those when $f_{m}=f_{r}$ but are larger than the case of $f_{m}=0.1 f_{r}$. The enhancement of harmonic distortions when $f_{m}=f_{r}$ was reported in $[16,17]$. Within the operating ranges of the period doubling effect, CPSPD and PPPD, HHD increases remarkably with the increase of $m$; it reaches levels much higher than those of both 2HD and 3HD. It is clear also that HHD is higher for the PPPD type than for the CPSPD type.

The corresponding variations of harmonic distortions with $m$ and $f_{m}$ when the non-modulated laser oscillates in two modes $\left(I=2.01 I_{t h}\right)$ are given in figure 7 . The figures show also that $2 \mathrm{HD}$ is higher than 3HD over the relevant ranges of $m$ and $f_{m}$. Figure 6(a) of $f_{m}=0.1 f_{r}$ indicates similar results to the case of the nearly single-mode oscillation of figure $6(\mathrm{a})$. When $f_{m}=f_{r}$, figure $7(\mathrm{~b})$ indicates that $2 \mathrm{HD}$ attains values smaller than those when $I_{b}=3 I_{t h}$, however it varies with $m$ in similar fashion. On the other hand, $3 \mathrm{HD}$ changes within the same range of the case of $I_{b}=3 I_{\text {th }}$ and in the same fashion except within the range $0.3>m>0.65$, where $3 \mathrm{HD}$ drops to $-37 \mathrm{~dB}$ when $m=0.5$ and then increases. The half harmonic distortion HHD has the same dependence on $m$ as the case of nearly single-mode oscillation. In the region of higher modulation frequency, $f_{m}=1.4 f_{m}$, figure 7 (c) shows that the higher harmonics $2 \mathrm{HD}$ and $3 \mathrm{HD}$ and the half harmonic HHD have values little larger than those of the case of single-mode oscillation of figure 6(c), but they vary with $m$ in similar fashion.

\section{Conclusions}

We introduced intensive large-signal simulations of mode competition dynamics and signal distortions associated with sinusoidal modulation of semiconductor lasers. Both higher and half harmonic distortions were investigated for both cases of nearly single mode oscillation and two-mode oscillations. Basing on the obtained results, the following conclusions are traced.

1. The modulated laser waveform is classified into three types of continuous variation with time; namely CPS, CPSRO and CPSPD, and three pulsing types; namely, PP, PPRO and PPPD. The 
classification is independent of the modal oscillation of the laser. The CPS type dominates the range of low values of $m$. When $f_{m}$ is low, the increase in $m$ converts the CPS type into the CPSRO type and then to the PPRO type. When $f_{m}$ is around $f_{r}$, the laser output is uniform, either the CPS type or PP type. When $f_{m} / f_{m}$ is large with $m>1.2$, the CPS and PPPD types dominates.

2. Both single and two mode lasers oscillate in single mode when the signal is continuous, whereas the pulsing signals are characterized by multimode oscillation. This range of the modulation depth of modulated single-mode oscillation is smaller for the two-mode non-modulated laser than for the nearly single-mode laser. Modulation of the two-mode laser is associated with wider operating ranges of the pulsing types than those of the single-mode laser.

3. The 2HD values of the nearly single-mode non-modulated laser are smaller than those of the twomode laser. HHD is enhanced when the modulated signal exhibits period doubling, increasing with the increase of the modulation depth. The higher harmonics are enhanced when the laser is modulated around the resonance frequency. The higher harmonic distortions are highest when the laser output is pulsating and the pulses are superposed by relaxation oscillations.

\section{References}

[1] C. Cox , Analog Optical Links Theory and Practice (Cambridge University Press, 2004).

[2] M Ahmed and M Yamada, IEEE J Quantum Electron. 38, 682 (2002).

[3] M. Ahmed, Physics D 176, 212 (2003).

[4] M. Yamada, IEEE J Quantum Electron QE-22, 1052 (1986).

[5] K. Y. Lau, C. M. Gee, T. R. Chen, N. Bar-Chaim and I. Ury, J. Lightwave Technol. 11, 1216 (2993).

[6] M. Yamada, J. Appl. Phys. 66, 81 (1989).

[7] N. Ogasawara and R. Ito, Jpn. J. Appl. Phys., 27607 (1988).

[8] M. Yamada, W. Ishimori, H. Sakaguchi and M. Ahmed, IEEE J Quantum Electron 39, 1548 (2003).

[9] M. Ahmed and A. El-Lafi, Opt. Laser Technol. 40, 809 (2008).

[10] M. Ahmed and M. Yamada, submitted to J. Appl. Phys. 
[11] M. Ahmed, J. Phys. D 41, 175104-1 (2008).

[12] K. Y. Lau, C. Harder and A. Yariv, IEEE J. Quantum Electron. QE-20, 71 (1984).

[13] R. V. Dalal, Investigation of High Linearity DFB Lasers for Analog Communications, Master Thesis, Massachustts Institute of Technology, USA, 1998.

[14] J. B. Song and A. H. M. R. Islam, J. Lightwave Technol. 26, 467 (2008).

[15] K.E. Stubkjr, “Nonlinearity of D.H. GaAlAs lasers,” Electron. Lett., Vol. 15, pp. 61-63, 1979.

[16] Y. Kam, and A. Yariv, Opt. Commun. 34, 424 (1980).

[17] M Ahmed, M Yamada and S Abdulrhmann, Fluct. Noise Lett. 1, L163 (2001).

[18] E. Henery, L. Chusseau and J. M. Lourtioz, IEEE J Quantum Electron. 26, 633 (1990).

[19] C. H. Lee, T. H. Yoon and S. Y. Shin, Appl Phys Lett. 46, 95 (1984).

[20] L. Chusseau, E. Henery and J. M. Lourtioz, Appl Phys Lett. 55, 822 (1989).

[21] M. Ahmed, N. Z. El-sayed and H. Ibrahim, Europ. J. Phys. D, (2012), in press.

[22] M. Ahmed and A. El-Lafi, Pramana J. Phys. 71, 90 (2008).

[23] G. P. Agrawal and N. K. Dutta, Semiconductor Lasers (New York: Van Nostrand Reinhold, 1993).

[24] Y. H. Kao and H. T. Lin, Phys. Rev. A 48, 2292 (1993). 


\section{Figure Captions}

Figure 1. Simulation characteristics of the non-modulated laser diode: (a) and (b) time variation of $S_{p}(t)$ of the strongest modes, (c) and (d) modal gain and intensity spectrum, (d) and (e) RIN spectra of the total output and strongest modes, when $I=2.76$ and $3.0 I_{t h}$, respectively.

Figure 2. The spectra of the linear gain $A_{p}$ and suppressed gain $G_{p}$ of the oscillating modes when $\mathrm{I}=2.01 I_{t h}$ (single mode oscillation) and 3.0I $I_{\text {th }}$ (single mode oscillation). The figure shows the asymmetric character of the suppressed gain

Figure 3. Characteristics of the waveform types of the modulated signal: (a) - (d) CPS type, (e) - (h) CPSRO type, (i) - (l) CPSPD type, (m) - (p) PP type, (q) - (t) PPRO type, and (u) - (x) PPPD type. The left hand side column plots the temporal trajectory of $S(t)$, the next column plots the transients of the strongest three oscillating modes, the third column plots the output spectrum of mode intensities, while the right hand side column plots the FFT power spectrum $S_{f}$.

Figure 4. ( $m$ versus $f_{m} / f_{r}$ ) diagram of the types of the modulated laser signal when $I_{b}=3 I_{t h}$ (single-mode laser). A contour plot of SMSR is included.

Figure 5. ( $m$ versus $f_{m} / f_{r}$ ) diagram of the types of the modulated laser signal when $I_{b}=2.01 I_{t h}$ (two-mode laser). A contour plot of SMSR is included.

Figure 6. Variations of harmonic distortions of the modulated signal when (a) $f_{m}=0.1 f_{r}$, (b) $f_{m}=f_{r}$, and (c) $f_{m}=1.4 f_{r}$, for $I_{b}=3.0 I_{t h}$ (nearly single-mode oscillation). The types of the modulated signal are written within the corresponding operating ranges of $m$.

Figure 7. Variations of harmonic distortions of the modulated signal when (a) $f_{m}=0.1 f_{r}$, (b) $f_{m}=f_{r}$, and (c) $f_{m}=1.4 f_{r}$, for $I_{b}=2.01 I_{t h}$ (two-mode oscillation). The types of the modulated signal are written within the corresponding operating ranges of $m$.

\section{Table Caption}

Table 1. Definitions and values of the parameters of the AlGaAs laser and fiber link used in the present calculations. 
Table 1

\begin{tabular}{|c|c|c|c|}
\hline Symbol & Meaning & Value & Unit \\
\hline \multicolumn{4}{|c|}{ Laser parameters } \\
\hline$\lambda$ & Emission wavelength & 850 & $\mathrm{~nm}$ \\
\hline$a$ & Tangential gain coefficient & $2.75 \times 10^{-12}$ & $\mathrm{~m}^{3} \mathrm{~s}^{-1}$ \\
\hline$\xi$ & Field confinement factor in the active layer & 0.2 & --- \\
\hline$b$ & $\begin{array}{c}\text { dispersion parameter of the linear gain } \\
\text { spectrum }\end{array}$ & $3 \times 10^{19}$ & $\mathrm{~m}^{3} \mathrm{~A}^{-2}$ \\
\hline$\alpha$ & Linewidth enhancement factor & 2.6 & -- \\
\hline$V$ & Volume of the active region & $150 \times 10^{-18}$ & $\mathrm{~m}^{3}$ \\
\hline$N_{g}$ & Electron number at transparency & $2.1 \times 10^{8}$ & --- \\
\hline$\delta \lambda$ & half-width of spontaneous emission & 23 & $\mathrm{~nm}$ \\
\hline$\tau_{\mathrm{s}}$ & Spontaneous emission lifetime & $2.79 \times 10^{-9}$ & $\mathrm{~s}$ \\
\hline$\left|R_{c v}\right|^{2}$ & Squared absolute value of the dipole moment & $2.8 \times 10^{-57}$ & $\mathrm{C}^{2} \mathrm{~m}^{2}$ \\
\hline$\tau_{\text {in }}$ & electron intraband relaxation time & 0.1 & ps \\
\hline$N_{s}$ & $\begin{array}{l}\text { Electron number characterizing gain } \\
\text { suppression }\end{array}$ & $1.7 \times 10^{8}$ & --- \\
\hline$L_{D}$ & Length of the active region & 300 & $\mu \mathrm{m}$ \\
\hline$n_{D}$ & Refractive index of the active region & 3.6 & - \\
\hline$R_{f}$ & Reflectivity of front facet & 0.3 & -- \\
\hline$R_{b}$ & Reflectivity of back facet & 0.6 & -- \\
\hline$G_{t h}$ & Threshold gain level & $3.22 \times 10^{11}$ & $\mathrm{~s}^{-1}$ \\
\hline$I_{\text {th }}$ & Threshold current & 15.4 & $\mathrm{~mA}$ \\
\hline
\end{tabular}


Figure 1

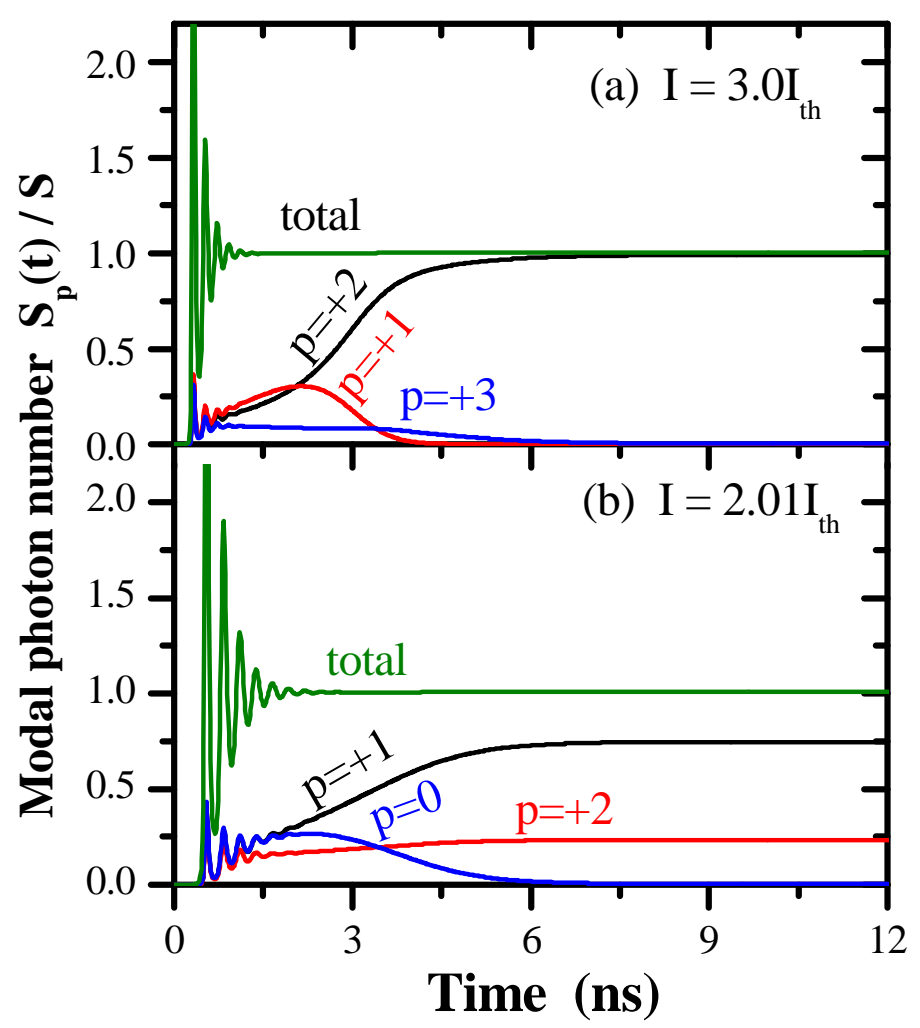


Figure 2

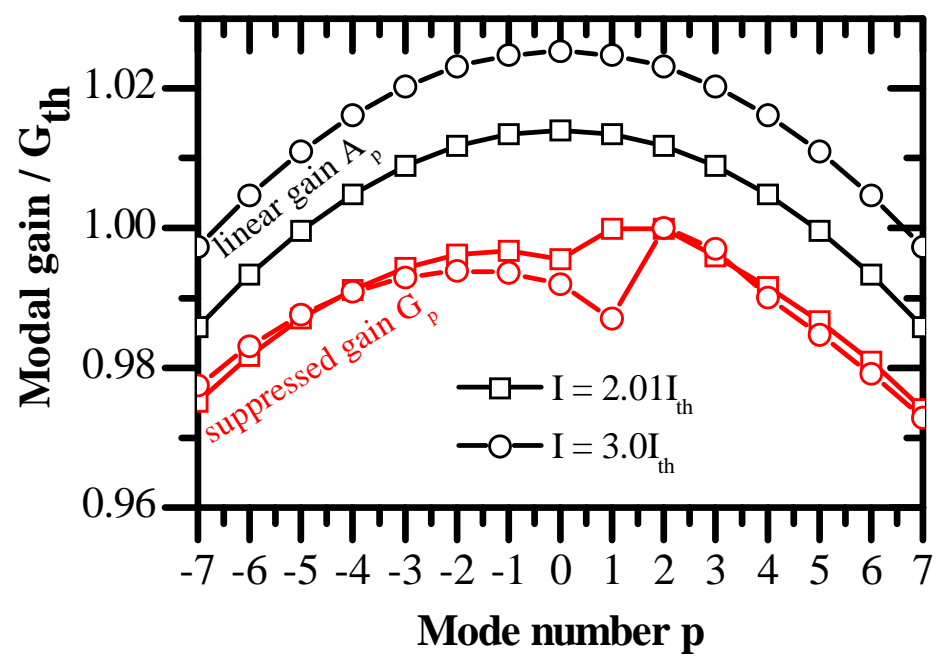


Figure 3
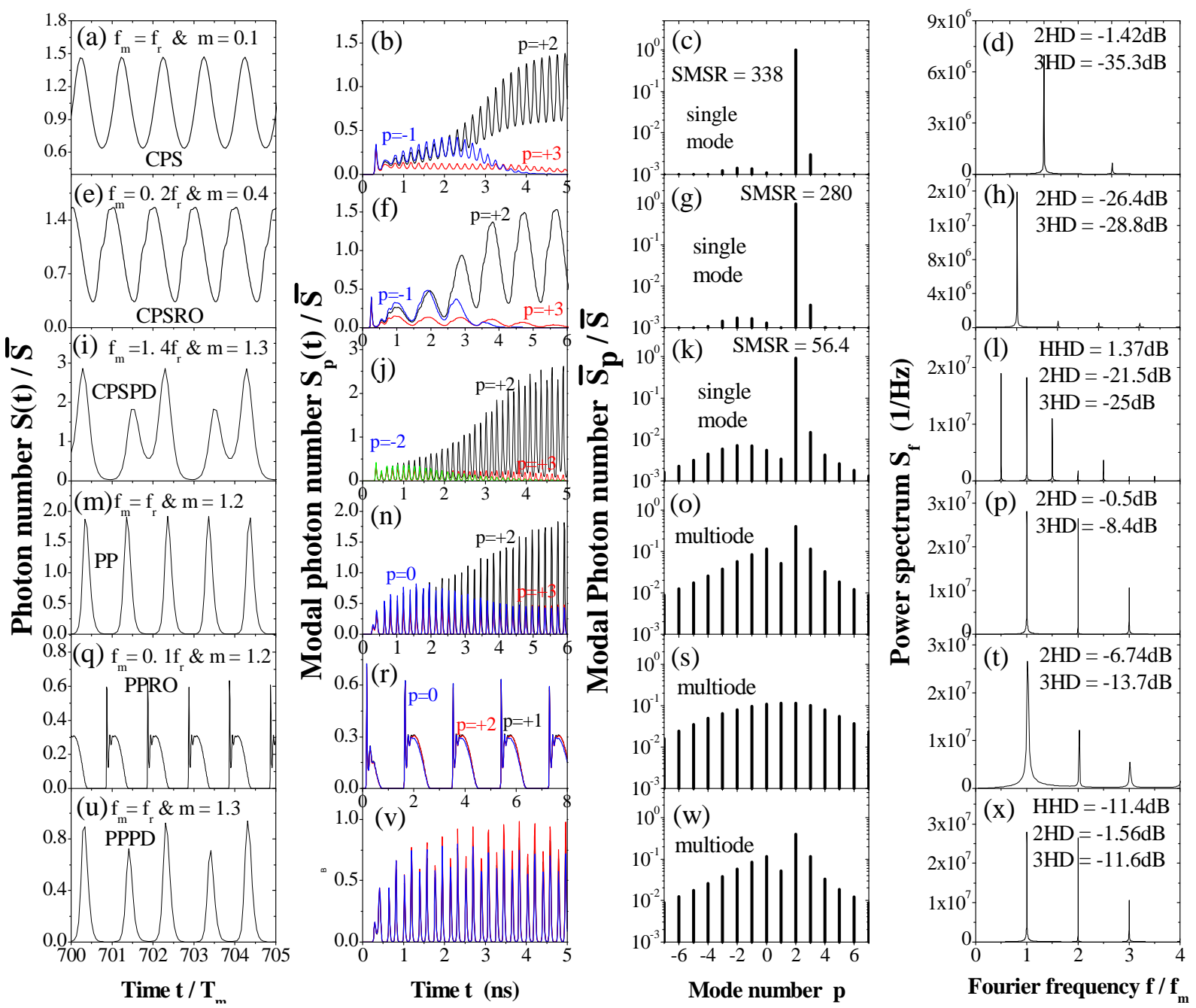
Figure 4

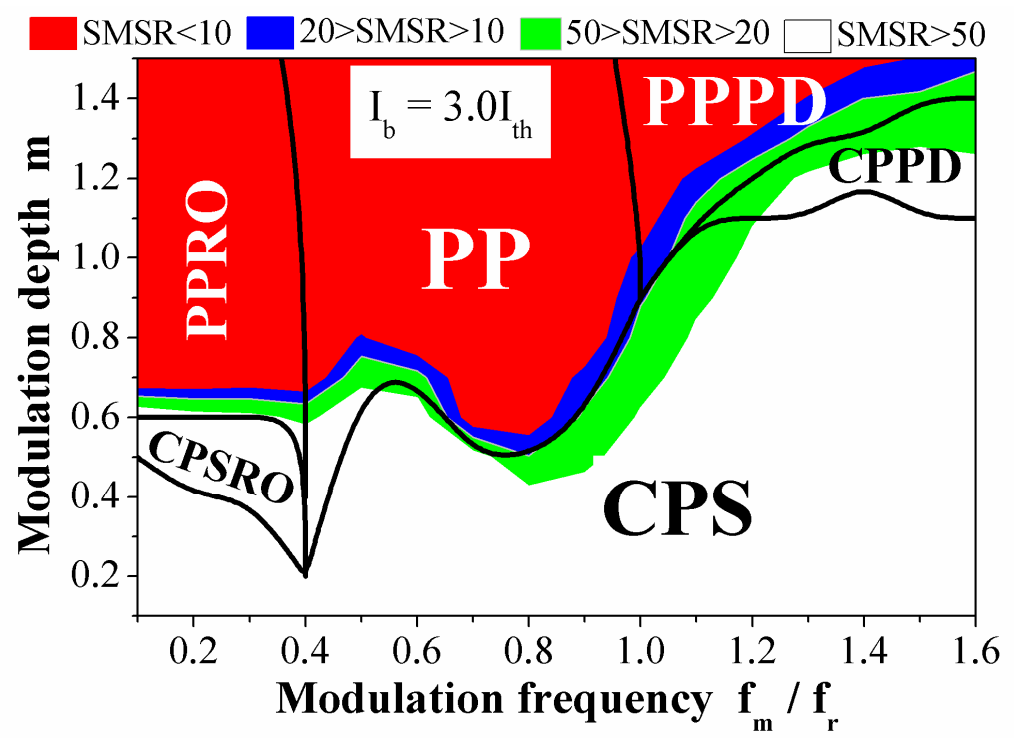


Figure 5

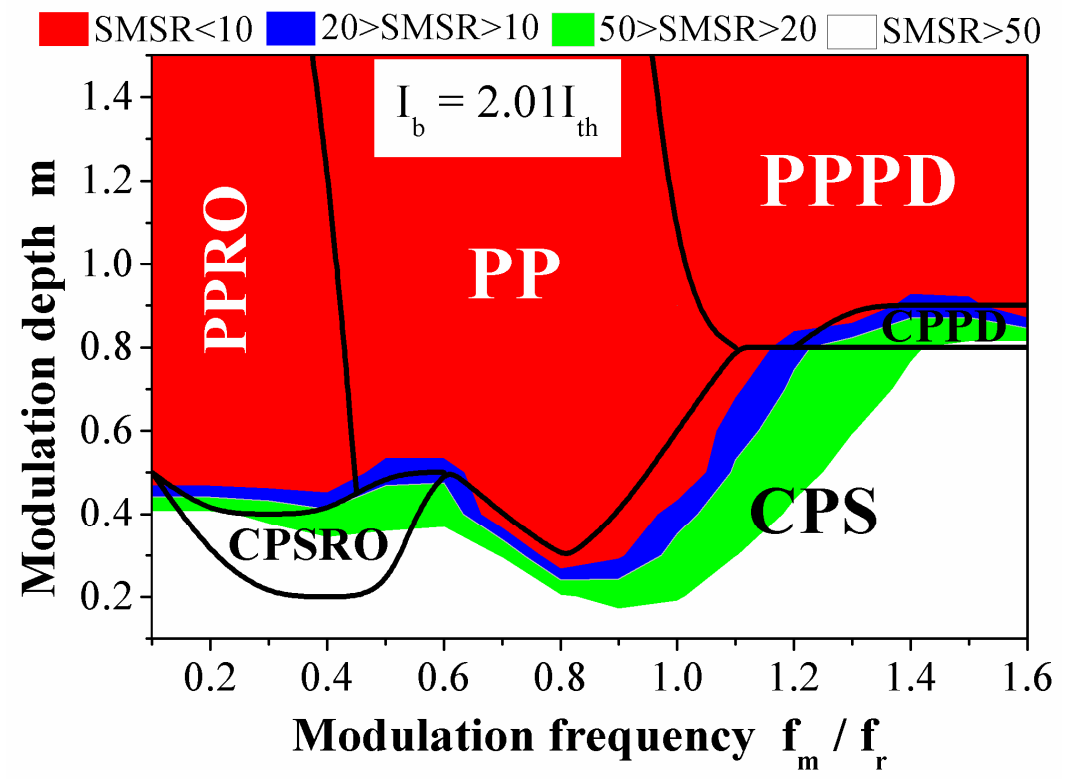


Figure 6

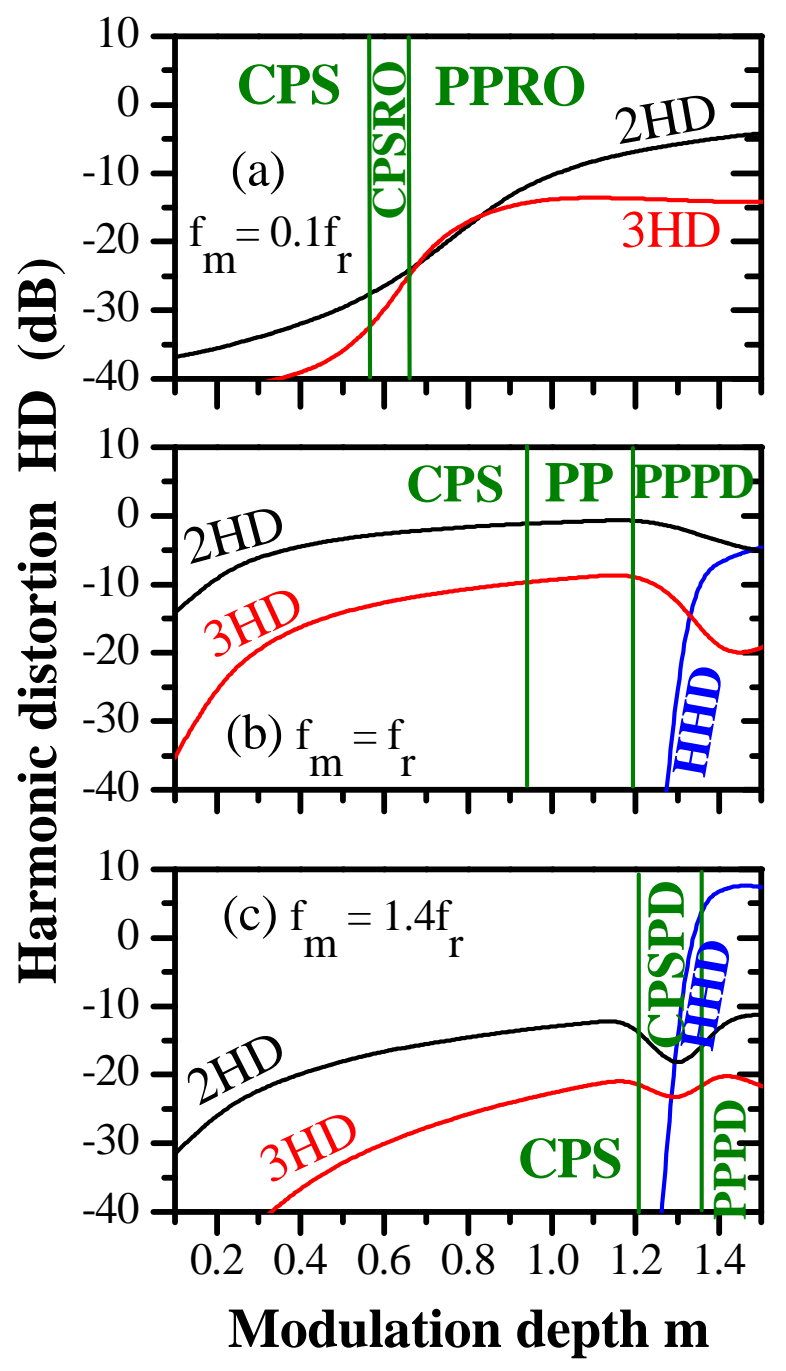


Figure 7

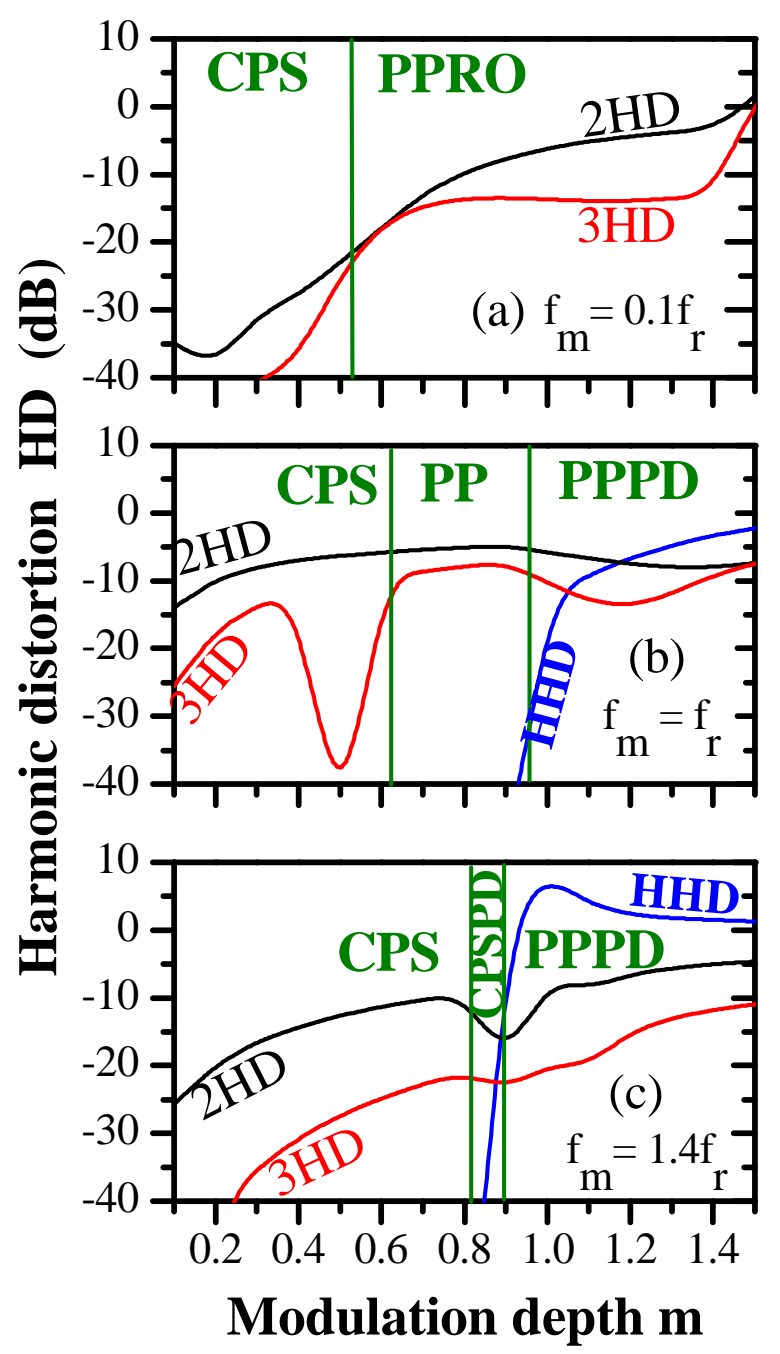

